\title{
Rapid Trajectory Optimization For the ARES I Launch Vehicle
}

\author{
Greg A. Dukeman* \\ NASA Marshall Space Flight Center, Huntsville, Alabama, 35812, USA \\ Ashley D. Hill ${ }^{\dagger}$ \\ Dynamic Concepts Incorporated, Huntsville, Alabama, 35812, USA
}

\begin{abstract}
A simplified ascent trajectory optimization procedure has been developed with application to NASA's proposed Ares I launch vehicle. In the interest of minimizing bending loads and ensuring safe separation of the first-stage solid rocket motor, the vehicle is constrained to follow a gravity-turn trajectory. This reduces the design space to just two free parameters, the pitch rate after a short vertical rise phase to clear the launch pad, and initial launch azimuth. The pitch rate primarily controls the in-plane parameters (altitude, speed, flight path angle) of the trajectory whereas the launch azimuth primarily controls the out-of-plane portion (velocity heading.) Thus, the optimization can be mechanized as two one-dimensional searches that converge quickly and reliably. The method is compared with POST-optimized trajectories to verify its optimality.
\end{abstract}

\section{Nomenclature}

$\begin{array}{ll}\text { DOLILU } & \text { Day-Of-Launch I-Load Update } \\ \text { ISS } & \text { International Space Station } \\ K S C & \text { Kennedy Space Center } \\ \text { MAVERIC } & \text { Marshall Aerospace Vehicle Representation in C } \\ M E C O & \text { Main Engine Cutoff } \\ \text { OTIS } & \text { Optimal Trajectories by Implicit Simulation } \\ \text { POST } & \text { Program to Optimize Simulated Trajectories } \\ S R B & \text { Solid Rocket Booster } \\ i & \text { orbital inclination } \\ \phi & \text { geocentric latitude } \\ \psi & \text { launch azimuth, positive clockwise from local North }\end{array}$

\section{Introduction}

An important ingredient of launch vehicle design and analysis and, ultimately, launch operations, is the capability to optimize ascent trajectories while satisfying constraints such as bending moments. These constraints are significantly influenced by wind conditions that exist in the region of maximum dynamic pressure. Plenty of software packages and procedures exist for performing trajectory optimization, including POST, ${ }^{1}$ OTIS, ${ }^{2}$ and DOLILU. ${ }^{3}$ POST and OTIS are very effective programs but are more powerful and generic than what is needed for Ares I. DOLILU is tailored to the space shuttle vehicle which is very different from the Ares I vehicle. The Atlas/Centaur launch vehicle trajectories are optimized using a software package called ADDJUST, ${ }^{4}$ which was developed after several early launch delays due to the winds aloft not fitting within the design wind envelopes. POST is the optimization package currently being used to generate Ares I design trajectories. The resulting optimal attitude profile is written to a file which is then manipulated

\footnotetext{
*Aerospace Engineer, Guidance, Navigation, and Mission Analysis Branch.

${ }^{\dagger}$ Senior Aerospace Engineer
} 
to extract the first-stage open-loop guidance I-loads. These I-loads are then uploaded to the computer that hosts the high-fidelity trajectory simulation, MAVERIC, being used for Ares I design and analysis.

The optimizer described in this paper is a quick and reliable optimizer that is integrated into MAVERIC. Such a capability enables efficient analysis of the potential benefits of day-of-launch optimization to prelaunch measured winds, versus optimization to, say, the month-of-launch mean KSC winds. It also serves as a pathfinder to the software and procedures to be used for Ares I day-of-launch operations.

The rest of this paper is organized as follows. The next section describes the Ares I trajectory sequence of events. The subsequent section describes the general optimization strategy which involves explicit numerical integration of first stage flight, while propagation and optimization of the upper stage flight phase takes advantage of efficient exo-atmopheric trajectory optimization. Next, numerical results involving comparisons with POST are given. The paper concludes with a summary and conclusions.

\section{Trajectory Sequence of Events}

On the launch pad, the vehicle is oriented so that the crew window points due East. After SRB (first-stage) ignition, the launch vehicle flies a vertical, stationary attitude until the vehicle has cleared the launch tower. This typically takes 6 seconds. Next, the vehicle commences a combined pitch/roll maneuver, continuing until the crew window is in the desired plane of the ascent trajectory. The vehicle pitches at a constant rate until the dynamic pressure builds up to 150 pounds per sq ft. Over the next 6 seconds, the vehicle transitions to the gravity turn condition, that is, total angle of attack of zero. The rest of first stage flight, until burn out of the SRB and separation, is flown in the gravity turn condition. This flight protocol keeps bending moments low and sets up a benign attitude (low aerodynamic forces and moments) and low angular rates (reduces lateral relative movement during separation) for the SRB separation sequence in which the interstage must clear the J2X engine nozzle. During upper stage flight, the vehicle flies a linear tangent steering profile, for optimum fuel usage, to a target orbit conic of prescribed orbit plane, cutoff altitude, apogee and perigee. Several seconds after upper stage flight commences, the Launch Abort System is jettisoned as are panels protecting the service module.

\section{Optimization Procedure}

The function to be maximized is total mass injected into the target orbit. Given a trial pair of pitch rate and launch azimuth, the function can be evaluated by first numerically propagating the vehicle's translational states from liftoff through SRB separation, consistent with the trajectory sequence of events described in the previous section. The final states from this flight phase are treated as initial conditions for upper stage flight. These states (position, velocity and mass), along with the target orbit parameters and J2X engine thrust and Isp, are provided as inputs to an efficient exoatmospheric guidance code. ${ }^{5}$ One of the natural by-products of the guidance code is the injected mass, which is taken to be the optimized injected mass, given the trial pitch rate and launch azimuth.

Moving up a level, a one-dimensional parameter optimizer is used to optimize first the pitch rate (given a first guess at an optimal azimuth) and then the launch azimuth (with the optimized pitch rate.) From experience, only a very narrow range of pitch rates need be considered in the optimization, say, $0.5 \mathrm{deg} / \mathrm{s}$ to $1.5 \mathrm{deg} / \mathrm{s}$. The first guess for the launch azimuth, $\psi$, is obtained from spherical geometry, given the target inclination, $i$, and launch (geocentric) latitude, $\phi$ :

$$
\sin \psi=\frac{\cos i}{\cos \phi}
$$

The final optimized launch azimuth will generally be offset a few degrees from this first guess due to the constraint of flying a gravity turn in the presence of winds and due to Earth rotation.

Note that because the roll component of the (post-vertical rise) pitch-roll maneuver has no effect on the translational trajectory, the roll need not be modeled during the iterations of the optimization. The requirement to roll is accommodated in flight by commanding the desired roll angle as soon as the vertical rise phase has completed. The onboard flight software will then automatically fly a combined pitch-roll maneuver.

The one-dimensional optimization routine used here is Brent's algorithm as described in Numerical Recipes. ${ }^{6}$ It is guaranteed to converge once the optimum has been bracketed. An efficient steepest de- 
scent approach is used whenever possible, otherwise a golden-section search is used.

\section{Numerical Results}

A mission to the ISS was optimized using the ("day-of-launch") optimization process described above. Results illustrate that the simplified optimizer performs quite well in obtaining a solution very near the optimized POST trajectory. Final mass to orbit was exactly the same. Figure 1 compares the resulting angle of attack profile from POST and from the day-of-launch optimization procedure. Figure 2 compares the altitude profiles, Figure 3 compares the ground track and Figure 4 compares the dynamic pressure profiles. All the plots confirm that the simplified optimizer does as well as the POST program.

Next, a day-of-launch scenario was simulated in which a launch minus 2 hour (L-2hr) smoothed measured wind profile was used in the optimization process to get a steering profile for first-stage open-loop guidance. The vehicle was then flown to a wind measured two hours later (near launch time.) The purpose of this is to show how day-of-launch trajectory optimization helps reduce load indicators such as the product of dynamic pressure and total angle of attack. Figure 5 shows the angle of attack profile. Figure 6 shows the product of dynamic pressure and total angle of attack. It can be seen that the load indicator is reduced from $2,500 \mathrm{lb} / \mathrm{sq}-\mathrm{ft}$ to $1,500 \mathrm{lb} / \mathrm{sq}-\mathrm{ft}$ by re-optimizing the first-stage attitude profile to the winds measured shortly before launch, in contrast to flying an attitude profile optimized to the mean winds of the month of launch. Updated results to include Monte Carlo results wherein first-stage attitude profile is optimized to a 2-hour before launch smoothed measured wind, flown to "2-hour later" wind, showing how much improvement dayof-launch re-optimization provides compared to optimizing the first-stage attitude profile to month-of-launch mean winds.

\section{Conclusion}

A simplified ascent trajectory optimization package, suitable for the Ares I launch vehicle, is presented. It is shown that the design space can be reduced to just two free parameters, an initial pitch rate that determines the in-plane trajectory characteristics, and the launch azimuth that determines the out-of-plane trajectory characteristics. Each of the two parameters influence separate subsets of the trajectory space so that they can be optimized separately in a one-two sequence of one-parameter optimizations. Each of the optimizations have well-defined optima, which allows for quick and reliable optimization. This new procedure is integrated into the high-fidelity trajectory simulation software being used for Ares I design and analysis. This offers a process improvement compared to performing an offline optimization in POST and subsequent transcribing of the POST outputs into a data format useable by the first-stage guidance in Maveric. Optimized trajectories using this new procedure compare favorably with corresponding optimized trajectories from POST.

\section{References}

\footnotetext{
${ }^{1}$ Brauer, G., Cornick, D., Habeger, A., and Peterson, F., "Program to Optimize Simulated Trajectories (POST). Volume 3: Programmer's manual," Final report, Martin Marietta Corp., Denver, CO, April 1975.

${ }^{2}$ Hargraves, C. R. and Paris, S. W., "Direct Trajectory Optimization Using Nonlinear Programming and Collocation," Journal of Guidance, Control, and Dynamics, Vol. 10, 1987, pp. 338-348.

3 "DOLILU II System Definition and Requirements Document, NSTS 083299, Volume VI, DOLILU II Quality Assurance Rules, Day-of-Launch Function, DIVDT Program," Tech. rep., National Aeronautics and Space Administration, Feb. 1992.

${ }^{4}$ Swanson, D., "ADDJUST-An Automated System for Steering Centaur Launch Vehicles in Mesured Winds," Proceedings of the Seventh Conference on Aerospace and Aeronautical meteorology, AIAA, Melbourne, FL, Nov. 1976.

${ }^{5}$ Dukeman, G. A., "Atmospheric Ascent Guidance for Rocket-Powered Launch Vehicles," Proceedings of the AIAA Guidance, Navigation, and Control Conference, AIAA, Wahington, D.C., 2002.

${ }^{6}$ Press, W., Teukolsky, S., Vetterling, W., and Flannery, B., Numerical Recipes in C: The Art of Scientific Computing, Cambridge University Press, 2nd ed., 1992.
} 


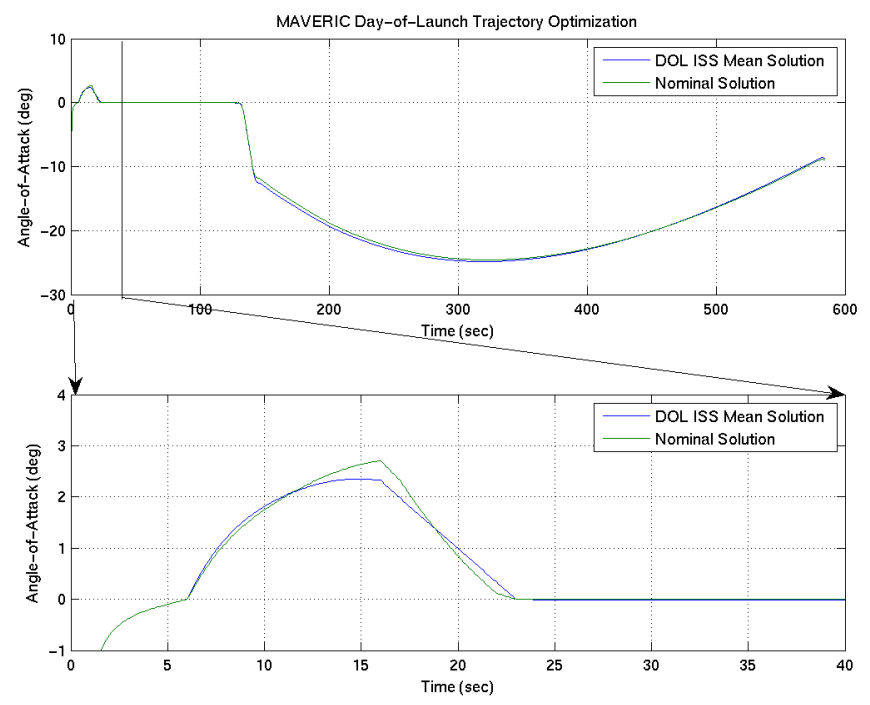

Figure 1. ISS Mission Angle Of Attack Profile.

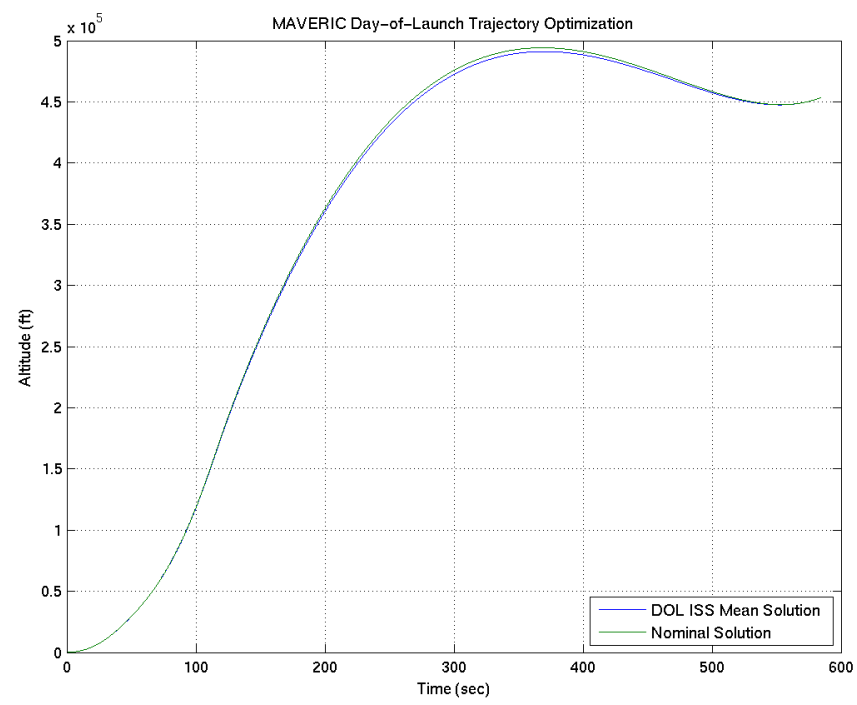

Figure 2. ISS Mission Altitude Profile. 


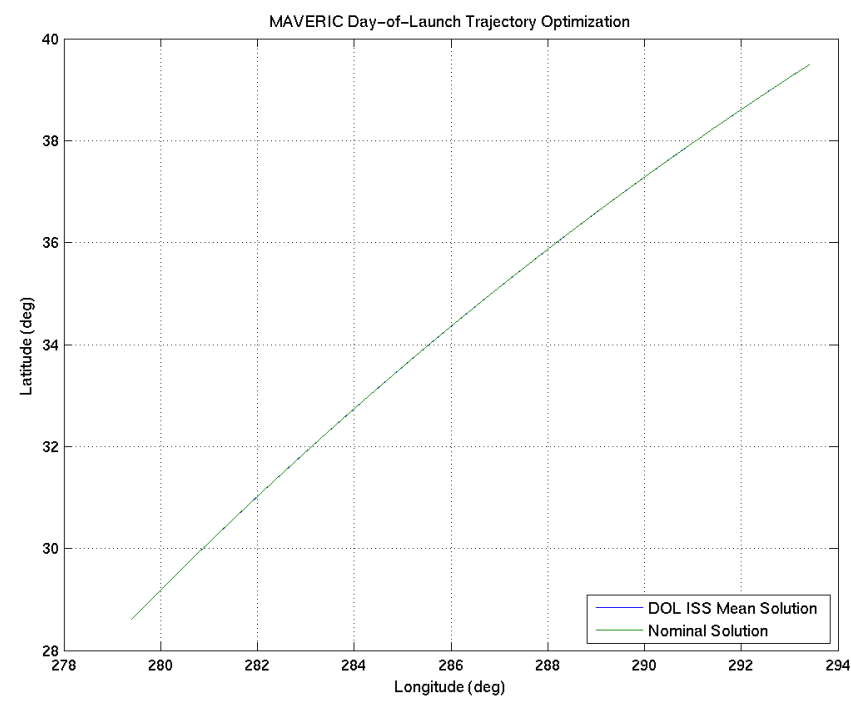

Figure 3. ISS Mission Ground Track.

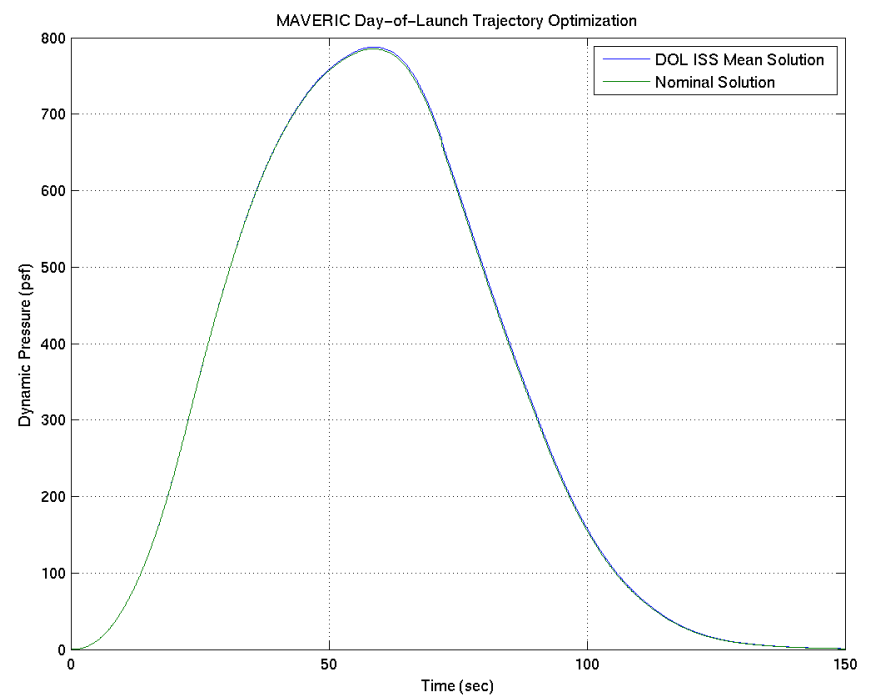

Figure 4. ISS Mission Dynamic Pressure Profile. 


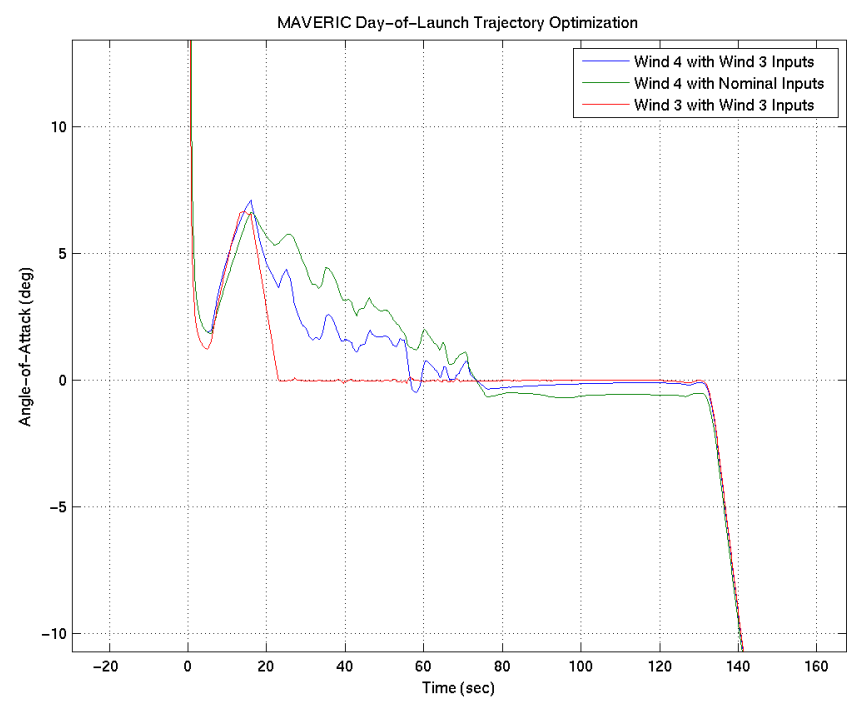

Figure 5. Angle of Attack Profile for Launch 2-hour Wind Pair.

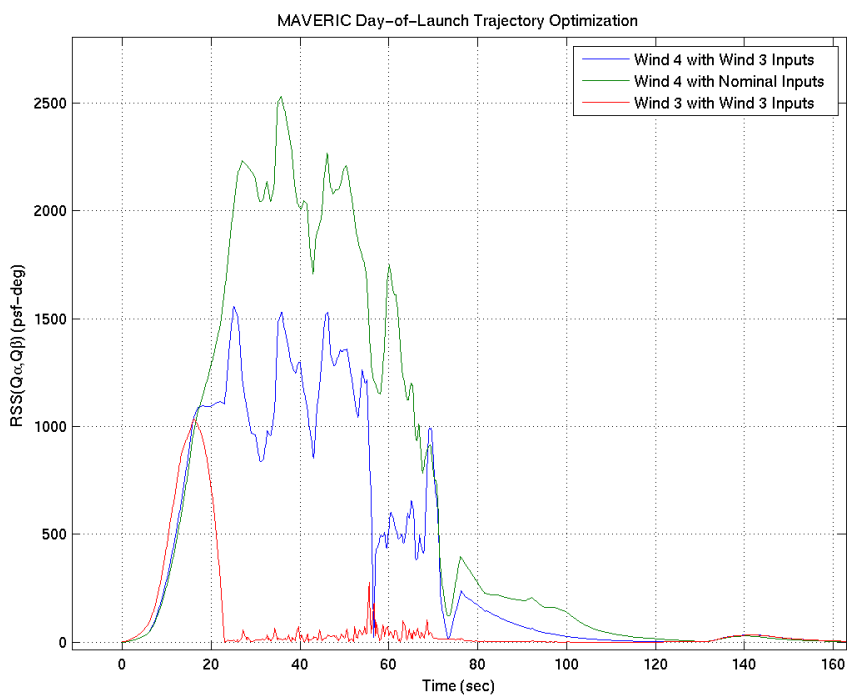

Figure 6. Product of Dynamic Pressure and Total Angle of Attack, Launch Wind Pair. 